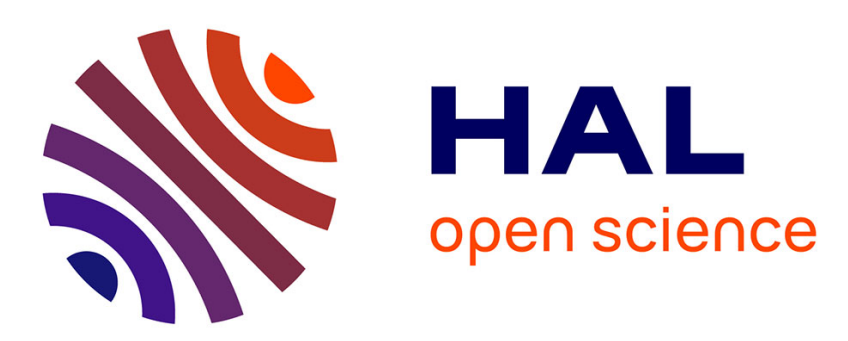

\title{
Analyse et classification d'exemples illustratifs dans le dictionnaire "Cesselin" en utilisant Google Traduction et un dictionnaire UNL-UWs
}

\author{
Mutsuko Tomokiyo, Mathieu Mangeot-Nagata, Christian Boitet
}

\section{- To cite this version:}

Mutsuko Tomokiyo, Mathieu Mangeot-Nagata, Christian Boitet. Analyse et classification d'exemples illustratifs dans le dictionnaire "Cesselin" en utilisant Google Traduction et un dictionnaire UNLUWs. 11es journées du réseau Lexicologie Terminologie Traduction 2018, Sep 2018, Grenoble, France. hal-01992882

\section{HAL Id: hal-01992882 \\ https://hal.science/hal-01992882}

Submitted on 24 Jan 2019

HAL is a multi-disciplinary open access archive for the deposit and dissemination of scientific research documents, whether they are published or not. The documents may come from teaching and research institutions in France or abroad, or from public or private research centers.
L'archive ouverte pluridisciplinaire HAL, est destinée au dépôt et à la diffusion de documents scientifiques de niveau recherche, publiés ou non, émanant des établissements d'enseignement et de recherche français ou étrangers, des laboratoires publics ou privés. 


\title{
Analyse et classification d'exemples illustratifs dans le dictionnaire "Cesselin" en utilisant Google Traduction et un dictionnaire UNL-UWs
}

\author{
Mutsuko Tomokiyo, Mathieu Mangeot-Nagata, Christian Boitet \\ LIG-GETALP, Bâtiment IMAG, 700 avenue Centrale F-38400 St. Martin d'Hères, France \\ mutsuko.tomokiyo@imag.fr, Mathieu.Mangeot@imag.fr, Christian.Boitet@imag.
}

\begin{abstract}
Résumé
Savoir si un exemple dans un dictionnaire bilingue est un proverbe, une expression idiomatique, une collocation ou un simple exemple d'utilisation serait très utile aux utilisateurs, mais est rarement indiqué. Dans cet article, nous présentons une classification manuelle et une annotation d'exemples dans un dictionnaire bilingue japonaisfrançais, le «Cesselin » (145000 entrées), dérivées de l'analyse manuelle de 500 entrées, et décrivons les étapes d'automatisation du processus d'annotation. Quant aux proverbes, nous utilisons simplement des dictionnaires de proverbes. Pour les autres cas, notre méthode consiste à comparer la traduction (automatique) en anglais d'un exemple original (japonais) et sa traduction (française) dans le dictionnaire. La comparaison est basée d'abord sur le nombre de lexèmes communs aux deux sorties (anglaises), et deuxièmement sur le nombre de lexèmes synonymes, qui peuvent être trouvés en utilisant un grand dictionnaire UNL-UW. Nous montrons notre procédure de classification en détail et son automatisation future.
\end{abstract}

Mots-clés : classification des exemples dans un dictionnaire bilingue, annotation manuelle des exemples, automatisation de la classification des exemples, Cesselin, UNL-UW dictionary, Google Traduction

\section{Analysis and classification of illustrative examples in the dictionary "Cesselin" by using Google Translation and UNL-UWs dictionary}

Knowing whether an example in a bilingual dictionary is a proverb, an idiomatic expression, a collocation, or a simple usage example would be quite helpful to users, but is rarely indicated. In this paper, we present a manual classification and annotation of examples in a Japanese-French bilingual dictionary, the "Cesselin” (145000 entries), derived from the manual analysis of 500 entries, and outline steps towards automating the annotation process. As for the proverbs, we simply use proverb dictionaries. For the other cases, our method relies on comparing the (automatic) translation into English of an original (Japanese) example and its (French) translation in the dictionary. The comparison is based first on the number of common lexemes in the two (English) outputs, and second on the number of synonymous lexemes, which can be found using a large UNL-UW dictionary. We show our classification procedure in detail and its future automatization.

\section{Google翻訳とUNL-UWs辞書を使った和仏大辞典 Cesselinの例文分析とその分類}

Keywords: classification of examples in a bilingual dictionary, annotation of examples, automatization of the classification, Cesselin jp-fr dictionary, UNL-UW dictionary, Google translation

\section{Introduction}

Nous présentons des travaux en cours sur un dictionnaire bilingue japonais-français, le "Cesselin" ${ }^{1}$, qui a été publié en 1939 et 1957 au Japon. Notre objectif est de le rendre disponible en tant que dictionnaire en ligne pour les apprenants de japonais ou de français, les chercheurs en études japonaises et les développeurs de systèmes de traduction automatique japonais-français (Mangeot, 2016).

1 和仏大辞典 (Wafutsu daijiten, Grand dictionnaire japonais-français), Cesselin, Gustave Jean Baptiste Cesselin, 丸善 (Maruzen) et 三省堂 (Sanseidô), 1939, Japon. 
Le dictionnaire Cesselin (Cesselin) inclut des connaissances sur la langue japonaise parlée et écrite ${ }^{2}$ et de riches exemples illustratifs des mots-vedette, tels que des descriptions phonétiques aujourd'hui obsolètes, les anciens caractères chinois et l'ancienne façon d'écrire les Okurigana ${ }^{3}$. Il n'est cependant pas satisfaisant que les exemples soient donnés sans indication ${ }^{4}$ concernant leur nature : sont-ils des proverbes, des exemples d'utilisation normale, des exemples de quantificateurs, ou d'autres types de collocations ? Leur introduction permettrait aux développeurs de systèmes de TA de traiter méthodiquement les ambiguïtés lexicales (Tomokiyo, Boitet \& Mangeot, 2017). Cela aiderait aussi les apprenants ou les chercheurs en langues à pratiquer le japonais ou le français et à approfondir leur compréhension de la culture japonaise.

Après avoir analysé un ensemble de 500 exemples (sur 280000 exemples estimés), nous avons défini une classification des exemples et un processus de classification. La prochaine étape envisagée est de l'automatiser pour économiser le plus de temps d'expert humain possible.

Dans la section 2, nous rapportons une expérience faite afin d'étudier quels types d'exemples sont inclus dans le Cesselin. Dans la section 3, nous proposons une classification, et dans la section 4, une procédure de classification d'un exemple donné. Après avoir expliqué le dictionnaire d'UWs dans la section 5 , nous proposons une procédure de classification automatique et examinons quelques aspects techniques de son automatisation dans la section 6 .

\section{2 Études de cas utilisant le système Google Translation pour la classification.}

Pour classer tous les exemples dans le Cesselin en fonction de leurs usages linguistiques, nous avons traduit 500 exemples japonais et leurs traductions françaises en anglais en utilisant le système de traduction de Google ("GT" ci-dessous), et avons comparé les deux traductions.

Notre approche est basée sur l'hypothèse que, si un exemple est un proverbe, ou si un mot dans un exemple est utilisé dans un usage collocatif, les lexèmes dans l'exemple traduit JE devraient différer de ceux de l'exemple traduit FE, parce que, dans les proverbes et les expressions collocatives, y compris les classificateurs, les mots semblent avoir tendance à être utilisés au sens figuré (Tomokiyo, Boitet \& Mangeot, 2017), et GT ne fournit pas de traductions adéquates pour eux (Tomokiyo \& Boitet, 2016), mais plutôt traductions word-to-word. Ainsi, les deux sorties contiennent des lexèmes différents, et on peut distinguer les proverbes et les collocations des exemples d'utilisation ordinaires.

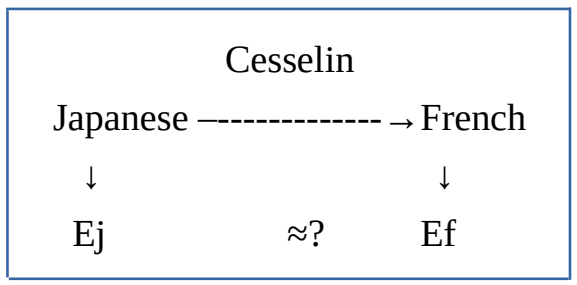

Figure 1: Hypothèse: $\mathrm{r}(\mathrm{Ej}) \subseteq \subseteq_{-} \mathrm{r}(\mathrm{Ef}) \approx=$ usage collocatif, proverbial, idiomatique

2 Le système d'écriture japonais a été fixé par le gouvernement japonais en 1986, où la façon d'écrire est conforme à la façon de prononcer.

3 Un mot japonais est écrit en Kanji (漢字, caractère chinois) et Hiragana (平仮名, caractère japonais), en kanji seulement, en hiragana seulement ou en katakana (片仮名). Les okuriganas (送り仮名) sont des suffixes qui suivent suivant les racines écrites en kanji. Les règles pour les okuriganas ont changé plusieurs fois, et ont été standardisées par le gouvernement japonais en 1973.

4 À l'exception des indications relatives à l'utilisation d'un sens figuré, par l'étiquette \{fig\}, et à l'utilisation de noms de domaine des mots-vedette. 


\subsection{Traduction japonais-anglais et français-anglais de $\mathbf{5 0 0}$ exemples par GT}

La description dans le Cesselin est structurée pour une entrée comme: mot-vedette (en japonais), prononciation, donnée avec deux formes différentes d'écriture (en japonais et en translittération en caractères latins, appelée ロマ字 (Romaji)), les formes conjuguées pour les verbes et les adjectifs, la partie du discours (POS), des définitions (en français) et des exemples en japonais avec leur translittération en romaji, et une traduction ou des traductions en français.

Exemple : Extrait du verbe "nomu (drink)" du Cesselin

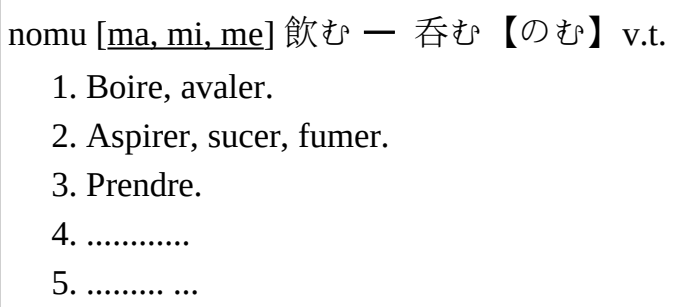

3. Prendre.

4.

5.

飲まねば薬も効能なし (Nomaneba kusuri mo kônô nashi) "Qui veut la fin veut les moyens." 飲まぬ酒には酔はぬ (Nomanu sake ni wa yowanu) "II n'y a point de fumée sans feu." 飲めや唄へや (Nome ya utae ya) "Faisons la noce et soyons joyeux!" ..........

Nous avons soumis à GT 500 exemples (contenus dans une vingtaine de mots-vedette) sélectionnés dans le Cesselin (voir les colonnes (a) et (d) dans le Tableau 1): 飲む(nomu, boisson), 買う (kau, acheter), 走る(hashiru, ran), 居る (iru, rester, être), 来る(kuru, viens), 思う (omou, penser), 言う (iu, say), 可愛い (kawaii, jolie), 強い (tsuyoi, fort), 安い (yasui, bon marché), 直ぐ (sugu, bientôt, immédiatement), から (kara, parce que, depuis, depuis), 家 (ie, maison, famille), 夜 (yoru, nuit), 足 (ashi, jambe), 車 (kuruma, voiture), 年 (nen, année), なん時 (nanji, à quelle heure), だけ (dake, seulement), ながら (nagara, while), 必要 (hitsuyou, nécessité), もう (mou, déjà), 事(koto, chose). Nous avons utilisé GT pour produire des traductions du japonais vers l'anglais et du français vers l'anglais (voir les colonnes (b) et (c) du Tableau 1).

\begin{tabular}{|c|c|c|c|c|c|}
\hline & (a) & (b) & (c) & (d) & (e) \\
\hline & $\begin{array}{l}\text { Exemples japonais } \\
\text { du Cesselin }\end{array}$ & $\begin{array}{l}\text { J-E } \\
\text { traductions par GT }\end{array}$ & $\begin{array}{l}\text { F-E } \\
\text { traductions par GT }\end{array}$ & $\begin{array}{c}\text { Traductions } \\
\text { françaises dans le } \\
\text { Cesselin }\end{array}$ & $\begin{array}{l}\text { Correspondant } \\
\text { aux résultats pour } \\
\text { J-E et F-E }\end{array}$ \\
\hline 1 & $\begin{array}{l}\text { 飲まねば薬も効 } \\
\text { 能なし。 } \\
\text { (Nomaneba } \\
\text { kusurimo kounou } \\
\text { nashi.) }\end{array}$ & $\begin{array}{l}\text { Il n'y a pas } \\
\text { d'indication pour un } \\
\text { médicament si vous } \\
\text { ne buvez pas. }\end{array}$ & $\begin{array}{l}\text { Who wants the end } \\
\text { wants the means. }\end{array}$ & $\begin{array}{l}\text { Qui veut la fin veut } \\
\text { les moyens. }\end{array}$ & $\begin{array}{l}\emptyset^{5} \text { (dic. de } \\
\text { proverbes) }\end{array}$ \\
\hline 2 & $\begin{array}{l}\text { 飲まぬ酒には酔 } \\
\text { わぬ。(Nomanu } \\
\text { sake niha }\end{array}$ & $\begin{array}{l}\text { I'm not intoxicated } \\
\text { with drinking } \\
\text { alcohol. }\end{array}$ & $\begin{array}{l}\text { There is no smoke } \\
\text { without fire. }\end{array}$ & $\begin{array}{l}\text { II n'y a point de } \\
\text { fumée sans feu. }\end{array}$ & $\begin{array}{l}\varnothing \text { (dic. de } \\
\text { proverbes) }\end{array}$ \\
\hline
\end{tabular}

5 Zéro correspondance des mots

6 L'exemple figure dans le dictionnaire de proverbes. 


\begin{tabular}{|c|c|c|c|c|c|}
\hline & (a) & (b) & (c) & (d) & (e) \\
\hline & yowanu.) & & & & \\
\hline 3 & $\begin{array}{l}\text { 飲めや唄へやの } \\
\text { 大騒ぎ。 (Nome } \\
\text { ya etae ya no } \\
\text { oosawagi.) }\end{array}$ & $\begin{array}{l}\text { Drinking and singing } \\
\text { fudge fuss. }\end{array}$ & $\begin{array}{l}\text { Let's do the wedding } \\
\text { and be merry! }\end{array}$ & $\begin{array}{l}\text { Faisons la noce et } \\
\text { soyons joyeux! }\end{array}$ & $\begin{array}{l}\varnothing \\
\text { (collocation) }\end{array}$ \\
\hline 4 & $\begin{array}{l}\text { 飲むに減らず吸 } \\
\text { うに減る。 } \\
\text { (Nomuni ni herazu } \\
\text { suu ni heru.) }\end{array}$ & $\begin{array}{l}\text { Reduce to suck } \\
\text { without reducing to } \\
\text { drink. }\end{array}$ & $\begin{array}{l}\text { It is not by dint of } \\
\text { drinking, but by dint } \\
\text { of sucking that it } \\
\text { diminishes. }\end{array}$ & $\begin{array}{l}\text { Ce n'est pas à force } \\
\text { de boire, mais bien à } \\
\text { force de sucer que ça } \\
\text { diminue, }\end{array}$ & $\varnothing$ \\
\hline 5 & $\begin{array}{l}\text { 飲むに減らず吸 } \\
\text { うに減る。 } \\
\text { (Nomuni ni herazu } \\
\text { suu ni heru.) }\end{array}$ & $\begin{array}{l}\text { Reduce to suck } \\
\text { without being } \\
\text { reduced to drink. }\end{array}$ & $\begin{array}{l}\text { Les dépenses les plus } \\
\text { répétées, bien que peu } \\
\text { sensibles à chaque } \\
\text { occasion, restent les } \\
\text { plus préoccupantes. }\end{array}$ & $\begin{array}{l}\{\text { fig }\}^{7} \text { Les dépenses } \\
\text { les plus répétées, } \\
\text { quoique peu } \\
\text { sensibles à chaque } \\
\text { fois, restent les plus } \\
\text { inquiétantes. }\end{array}$ & $\begin{array}{l}\varnothing \text { (dic. de } \\
\text { proverbes) \{fig }\}\end{array}$ \\
\hline 6 & $\begin{array}{l}\text { 飲んだり吐出し } \\
\text { たりする。 } \\
\text { (Nondari } \\
\text { hakidashitari } \\
\text { suru.) }\end{array}$ & It drinks or spits out. & $\begin{array}{l}\text { Swallow and turn in } \\
\text { turn. }\end{array}$ & $\begin{array}{l}\text { Avaler et rendre tour } \\
\text { à tour, }\end{array}$ & $\varnothing$ \\
\hline 7 & $\begin{array}{l}\text { 飲んだり吐出し } \\
\text { たりする。 } \\
\text { (Nondari } \\
\text { hakidashitari } \\
\text { suru.) }\end{array}$ & It drinks or spits out. & Accept and refuse. & $\begin{array}{l}\{\text { fig }\} \text { Accepter et } \\
\text { refuser. }\end{array}$ & $\begin{array}{l}\varnothing\{\text { fig }\} \\
\text { (collocation) }\end{array}$ \\
\hline 8 & $\begin{array}{l}\text { 飲んで吐き出し } \\
\text { た様。(Non de } \\
\text { hakidashita you.) }\end{array}$ & $\begin{array}{l}\text { Like drinking and } \\
\text { spitting out. }\end{array}$ & $\begin{array}{l}\text { As if we had made } \\
\text { what we have just } \\
\text { swallowed. }\end{array}$ & $\begin{array}{l}\text { Comme si on avait } \\
\text { rendu ce que l'on } \\
\text { vient d'avaler }\end{array}$ & $\varnothing$ \\
\hline 9 & $\begin{array}{l}\text { 飲んで吐き出し } \\
\text { た様。(Non de } \\
\text { hakidashita you.) }\end{array}$ & $\begin{array}{l}\text { Like drinking and } \\
\text { spitting out }\end{array}$ & $\begin{array}{l}\text { avec a pale face and } \\
\text { suddenly swollen. }\end{array}$ & $\begin{array}{l}\text { \{fig\} Avoir une } \\
\text { figure pâle et comme } \\
\text { subitement } \\
\text { boursouflée. }\end{array}$ & $\begin{array}{l}\varnothing\{\text { fig\} } \\
\text { (collocation) }\end{array}$ \\
\hline 10 & $\begin{array}{l}\text { 飲んで悲しみを } \\
\text { 忘れる。(Non de } \\
\text { kanashimi wo } \\
\text { wasureru.) }\end{array}$ & $\begin{array}{l}\text { Drink and forget } \\
\text { sorrow. }\end{array}$ & $\begin{array}{l}\text { To drown his sorrows, } \\
\text { to leave his sorrows at } \\
\text { the bottom of the } \\
\text { glass. }\end{array}$ & $\begin{array}{l}\text { Noyer ses chagrins, } \\
\text { laissez ses peines au } \\
\text { fond du verre. }\end{array}$ & $\varnothing$ \\
\hline
\end{tabular}

Tableau 1: Extrait des résultats de l'expérience

7 \{fig\}: L'étiquette indiquant l'usage d'un sens abstrait, donnée dans les traductions en français d'exemples du Cesselin, même si la liste n'est pas exhaustive. Les étiquettes, (botanique), (zoologie), etc. sont données pour montrer le domaine d'utilisation du mot. 


\subsection{Comparaison et contraste des deux résultats de traduction}

La colonne (e) du tableau 1 montre une comparaison manuelle des sorties de la traduction J_E (colonne (b)) avec les traductions F_E (colonne (d)) du point de vue des lexèmes dans un exemple. Le tableau 2 montre le contraste des deux sorties de traduction.

\begin{tabular}{|c|c|c|}
\hline $\begin{array}{l}\text { 100\% de concordance (les résultats de la traduction J_E et de la traduction } \\
\text { F_E par GT sur cet exemple incluent exactement les mêmes mots) }\end{array}$ & $63 / 500$ & $12,6 \%$ \\
\hline $\begin{array}{l}100 \% \text { * (les résultats de la traduction J_E et de la traduction F_E par GT sur } \\
\text { cet exemple incluent des mots synonymes) }\end{array}$ & $79 / 500$ & $15,8 \%$ \\
\hline $\begin{array}{l}\text { La concordance des vocabulaires avec_ou__la traduction J_E est plus } \\
\text { longue que la traduction F_E, mais les deux traductions comprennent } \\
\text { presque les mêmes mots. De plus, la traduction F_E est plus longue que la } \\
\text { traduction J_E, mais les deux traductions contiennent presque les mêmes } \\
\text { mots.) }\end{array}$ & $26 / 500$ & $5,2 \%$ \\
\hline $\begin{array}{l}\text { Concordance au niveau de l'analyse morphosyntaxique (les résultats de la } \\
\text { traduction J_E et de la traduction F_E par GT sur cet exemple incluent les } \\
\text { mêmes mots pour le verbe prédicat et ses actants) }\end{array}$ & $15 / 500$ & $3,0 \%$ \\
\hline Pas de concordance ni d'expression collocative & $108 / 500$ & $21,6 \%$ \\
\hline$\varnothing\{$ fig\} : Pas de concordance ni d'expression collocative avec le label \{fig.\} & $8 / 500$ & $1,6 \%$ \\
\hline $\begin{array}{l}\varnothing: \text { Pas de concordance et pas d'expression collocative (la traduction J_E et } \\
\text { la traduction F_E par GT sur cet exemple n'incluent aucun mot commun.) }\end{array}$ & $161 / 500$ & $32.2 \%$ \\
\hline $\begin{array}{l}\text { Pas de concordance et il s'agit de proverbes (la traduction J_E et la } \\
\text { traduction F_E par GT sur cet exemple n'incluent aucun mot commun et } \\
\text { l'exemple japonais apparaît dans un dictionnaire de proverbes japonais }\end{array}$ & $32 / 500$ & $6,4 \%$ \\
\hline D : Pas de concordance avec le domaine d'utilisation & $4 / 500$ & $0,8 \%$ \\
\hline X : Zéro sortie par GT & $4 / 500$ & $0,8 \%$ \\
\hline
\end{tabular}

Tableau 2 : Comparaison entre les traductions JE et les traductions FE pour 500 exemples

D'après nos expériences, les exemples du Cesselin peuvent être classés en 5 classes : exemples d'usage ordinaires, proverbes, expressions collocatives, quantificateurs, et exemples spécifiques au domaine.

\section{Classer les exemples du Cesselin}

Dans les exemples suivants pour le verbe "飲む(nomu, boire)", (a) est un proverbe, (b) est une phrase incluant "飲む" dans un sens figuratif, et (c) est une phrase qui n'est pas un proverbe ni une collocation, mais un usage ordinaire. Quand on compare les lexèmes dans les traductions JE et FE par

8 http://kotowaza-allguide.com/ 
GT, alors que (a) et (b) ne contiennent aucun mot commun, en (c), les deux traductions ont deux mots ("boire" et "eau") en commun.

Cela confirme que les exemples montrant des usages ordinaires ont tendance à se traduire par la composition de mots communs. Nous avons pensé que ce phénomène pourrait être un atout pour classer tous les exemples du Cesselin, car les traductions japonais-anglais par GT sont presque des traductions mot à mot et, quand un mot dans les exemples japonais est utilisé dans le sens figuratif dans les contextes, les résultats de traduction ne correspondent jamais aux résultats de la traduction français-anglais.

\begin{tabular}{|l|l|l|l|l|}
\hline & Exemples japonais & Traductions JE & Traductions FE & \multicolumn{1}{c|}{$\begin{array}{c}\text { Traduction } \\
\text { d'exemples japonais }\end{array}$} \\
\hline $\begin{array}{l}\text { (a) } \\
\text { Proverbe }\end{array}$ & $\begin{array}{l}\text { 飲まぬ酒には酔わぬ9 } \\
\text { (nomanu sake ni-ha } \\
\text { yowanu) }\end{array}$ & $\begin{array}{l}\text { I'm not intoxicated } \\
\text { with drinking alcohol. }\end{array}$ & $\begin{array}{l}\text { There is no smoke } \\
\text { without fire. }\end{array}$ & $\begin{array}{l}\text { II n'y a point de } \\
\text { fumée sans feu. }\end{array}$ \\
\hline $\begin{array}{l}\text { (b) } \\
\text { Collocation }\end{array}$ & $\begin{array}{l}\text { 佊は妻くんに飲まれ } \\
\text { ている (Kare wa } \\
\text { saikun ni } \\
\text { nomareteiru) }\end{array}$ & $\begin{array}{l}\text { He is being drunk by } \\
\text { his wife. }\end{array}$ & is wife is bowing him. & Sa femme le berne. \\
\hline $\begin{array}{l}\text { (c) } \\
\text { Exemple } \\
\text { d'utilisation } \\
\text { ordinaire }\end{array}$ & $\begin{array}{l}\text { 水を飲む (Mizu wo } \\
\text { nomu) }\end{array}$ & Drink water. & Drink water. & Boire de l'eau. \\
\hline
\end{tabular}

Tableau 3: Exemples d'un proverbe, d'une expression collocative et d'un usage habituel

Parmi les exemples d'usage courant, les proverbes, les expressions collocatives, les quantificateurs et les exemples spécifiques au domaine, nous pouvons distinguer les proverbes d'autres types d'exemples en utilisant un dictionnaire de proverbes et en utilisant des labels tels que \{fig\} et \{botanic, zoology...\}, qui sont attachés à certains exemples dans le Cesselin, nous pouvons distinguer les expressions collocatives et les exemples spécifiques au domaine d'autres exemples, respectivement.

\section{Procédure de classification des exemples du Cesselin}

Nous proposons les étapes suivantes pour la classification des exemples.

\subsection{Différenciation des proverbes des expressions collocatives}

Étape 1) Nous distinguons les proverbes des expressions collocatives, en utilisant un dictionnaire de proverbes. Si un exemple apparaît dans le dictionnaire, nous le classons comme un proverbe.

Etape 2) Pour les expressions collocatives, si la traduction d'un exemple dans le Cesselin est marquée comme utilisation figurative (\{fig\}), nous la classons comme une expression collocative.

\begin{tabular}{|l|l|l|l|l|}
\hline & Exemples japonais & Traductions JE & Traductions FE & $\begin{array}{l}\text { Traduction française } \\
\text { d'exemples japonais }\end{array}$ \\
\hline $\begin{array}{l}\text { Collocation } \\
\text { sans }\end{array}$ & $\begin{array}{l}\text { 人を飲んでかかる } \\
\text { Hito wo non de }\end{array}$ & $\begin{array}{l}\text { I'm drinking people and } \\
\text { hanging. }\end{array}$ & $\begin{array}{l}\text { Miss someone, } \\
\text { look down. }\end{array}$ & $\begin{array}{l}\text { Manquer à quelqu'un, } \\
\text { regarder de haut. }\end{array}$ \\
\hline
\end{tabular}

9 Taduction mot-à-mot: On ne se saoule pas avec le Sake que l'on ne boit pas. 


\begin{tabular}{|c|c|c|c|c|}
\hline & Exemples japonais & Traductions JE & Traductions FE & $\begin{array}{l}\text { Traduction française } \\
\text { d'exemples japonais }\end{array}$ \\
\hline \multirow[t]{2}{*}{ indication } & (kakaru) $)^{10}$ & & & \\
\hline & 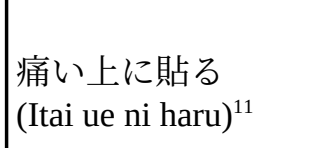 & Painfully stick it on & $\begin{array}{l}\text { misfortune on } \\
\text { misfortune. }\end{array}$ & malheur sur malheur. \\
\hline $\begin{array}{l}\text { Collocation } \\
\text { avec } \\
\text { indication } \\
\{\text { fig }\}\end{array}$ & $\begin{array}{l}\text { 飲んだり吐き出した } \\
\text { りする(Nondari } \\
\text { hakidashitari suru) }^{12}\end{array}$ & To drink or to spit. & Accept and refuse. & $\{$ fig\} Accepter et refuser. \\
\hline
\end{tabular}

Tableau 4: Exemples pour un mot ayant un sens figuré, avec ou sans l'étiquette \{fig\}

\section{2 Évaluation d'expressions collocatives sans étiquette dans le Cesselin}

Étape 3) Il y a des cas où les traductions françaises dans le Cesselin n'ont pas d'étiquette \{fig\} et, en l'état, elles sont utilisées au sens figuré. Si leurs traductions en anglais n'ont pas de lexème en commun $(r(E j) \cap r(E f)=\varnothing)$, nous les classons en tant qu'expressions collocatives.

\begin{tabular}{|l|l|l|l|l|}
\hline Mots-vedette & Exemples japonais & Traductions JE & Traductions FE & $\begin{array}{c}\text { Traduction en } \\
\text { français pour les } \\
\text { exemples japonais }\end{array}$ \\
\hline 言う (iu, say, tell) & $\begin{array}{l}\text { 言うに言われない } \\
\text { ほど (iu ni iware } \\
\text { nai hodo) }\end{array}$ & Not to be told to say & $\begin{array}{l}\text { Beyond any } \\
\text { expression. }\end{array}$ & $\begin{array}{l}\text { Au-delà de toute } \\
\text { expression. }\end{array}$ \\
\hline $\begin{array}{l}\text { 足 (ashi, jambe, } \\
\text { pied) }\end{array}$ & $\begin{array}{l}\text { arau })^{14} \\
\text { a (Ashi wo }\end{array}$ & Wash your feet & $\begin{array}{l}\text { Rise from a lower } \\
\text { class. }\end{array}$ & $\begin{array}{l}\text { S'élever d'une classe } \\
\text { inférieure. }\end{array}$ \\
\hline
\end{tabular}

Tableau 5: Exemples avec sens figuré sans aucune étiquette

Les deux traductions du tableau 5 n'ont pas de lexème en commun. Dans ce cas, la comparaison des exemples traduits est effectuée avec une liste KWIC (KeyWords In Context).

10 Traduction mot-à-mot : menacer quelqu'un en avalant.

11 Traduction mot-à-mot : coller quelque chose à la partie souffrante.

12 Traduction mot-à-mot : boire et cracher alternativement.

13 Traduction mot-à-mot : de façon à ne pas pouvoir dire si je dis.

14 Traduction mot-à-mot : se laver les pieds. L'expression 足を洗う a du sens en usage concret et en en même temps en usage figuré. Nous n'avons pas encore résolu ce problème. 


\subsection{Exemples traduits ayant une relation de synonymie avec des mots existants dans chaque exemple}

Étape 4) Dans le tableau 6, la traduction JE pour l'exemple 必要費est différente de la traduction FE pour "cost" et "expenses", et la traduction JE pour l'exemple 車 est différente de la traduction FE pour "ride" et "get in "のる", qui sont les verbes prédicatifs des phrases. Dans ces cas, la relation de synonymie entre les deux mots est examinée, et si les deux mots sont synonymes, l'exemple est classé comme exemple d'utilisation ordinaire.

\begin{tabular}{|c|c|c|c|c|c|}
\hline $\begin{array}{l}\text { Mots- } \\
\text { vedette }\end{array}$ & $\begin{array}{l}\text { Exemples } \\
\text { japonais }\end{array}$ & traduction JE & Traductions FE & $\begin{array}{l}\text { Traductions en } \\
\text { français }\end{array}$ & Comparaison \\
\hline $\begin{array}{l}\text { 必要 } \\
\text { (hitsuyou) }^{15}\end{array}$ & $\begin{array}{l}\text { 必要費16 } \\
\text { (hitsuyou hi) }\end{array}$ & Coût nécessaire & $\begin{array}{l}\text { Necessary } \\
\text { expenses. }\end{array}$ & $\begin{array}{l}\text { Dépenses } \\
\text { nécessaires. }\end{array}$ & $100 \% * 17$ \\
\hline $\begin{array}{l}\text { 車 } \\
\text { (Kuruma) }^{18}\end{array}$ & $\begin{array}{l}\text { 車に乗る } \\
\text { (kuruma ni } \\
\text { noru) }{ }^{19}\end{array}$ & I ride a car. & Get in the car & Monter en voiture. & $100 \% *$ \\
\hline
\end{tabular}

Tableau 6: Les deux exemples traduits qui ont des lexèmes synonymes

\subsection{Exemples traduits ayant une longueur différente}

Étape 5) Les exemples traduits en anglais dans le tableau 7 ont une longueur différente en raison de la nominalisation du verbe prédicatif, du degré différent de politesse des énoncés, etc. Dans ce cas, l'exemple est analysé : si le verbe prédicatif et son sujet (ou son objet) sont les mêmes, les deux exemples sont considérés comme ayant le même sens, et ils sont classés comme des exemples ordinaires.

\begin{tabular}{|c|c|c|c|c|}
\hline Mot-vedette & Exemples japonais & Traductions JE & Traductions FE & $\begin{array}{c}\text { Traduction en } \\
\text { français pour les } \\
\text { exemples japonais }\end{array}$ \\
\hline $\begin{array}{l}\text { もう (mou) } \\
\left(\mathrm{J} \_\mathrm{F}\right)\end{array}$ & $\begin{array}{l}\text { もう休みましょう } \\
\text { (Mou yasumi mashô) }\end{array}$ & Let's have a rest now. & Let's rest now! & $\begin{array}{l}\text { Reposons-nous. } \\
\text { maintenant! }\end{array}$ \\
\hline
\end{tabular}

15 Nécessaire.

16 Coût nécessaire.

$17100 \% *$ signifie que la correspondance des mots est $100 \%$ en utilisant la synonymie entre mots.

18 Voiture.

19 Monter en voiture.

20 Reposons-nous maintenant. 


\begin{tabular}{|c|c|c|c|c|}
\hline $\begin{array}{l}\text { ながら } \\
\text { (nagara) } \\
(\mathrm{J}+\mathrm{F})\end{array}$ & 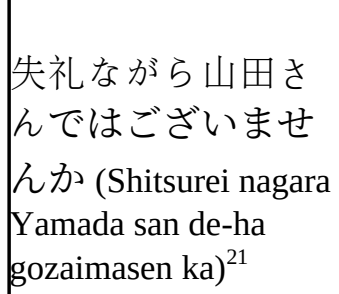 & $\begin{array}{l}\text { Excuse me, aren't } \\
\text { you Mr. Yamada? }\end{array}$ & $\begin{array}{l}\text { Please excuse me, } \\
\text { but aren't you Mr. } \\
\text { Yamada? }\end{array}$ & $\begin{array}{l}\text { Veuillez bien } \\
\text { m'excuser, mais } \\
\text { n'êtes-vous pas } \\
\text { monsieur Yamada? }\end{array}$ \\
\hline
\end{tabular}

Tableau 7: Deux exemples traduits de longueurs différentes

\section{Utilisation du dictionnaire de Mots Universels (UW) comme un pivot de lexèmes interlingues}

Afin d'obtenir des informations sur la synonymie et les relations morphosyntaxiques entre les mots dans les deux exemples traduits, nous avons utilisé un dictionnaire d'UWs du système de traduction automatique UNL ${ }^{22}$ comme un langage pivot interlingue. Un UW est une chaîne de caractères qui représente un sens de mot (renvoyant à un concept). Une représentation sémantique en UNL a la forme d'un hypergraphe, où les nœuds sont des UW (appelés Universal Words) ayant des attributs sémantiques, et les arcs portent des relations sémantiques ${ }^{23}$ entre deux UW. La description des mots est la suivante :

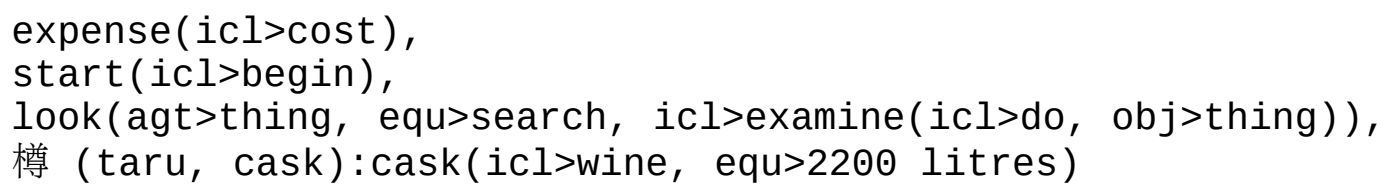

"agt" dénote que l'agent de "look" est "thing", et "look" appartient à un niveau sémantique équivalent dans la "préontologie » UNL ${ }^{24}$ avec "search", et inclut le sens de "examiner", "examiner" étant un verbe d'action et son objet grammatical un nom qui signifie "chose" (Uchida, Zhu et Dellasenta, 2006).

\section{Vers une classification automatique}

Pour effectuer une classification automatique des exemples, nous avons besoin d'un dispositif qui associe des phrases, des mots ou des lexèmes et analyse les exemples. Il passe par les étapes suivantes, suivant l'analyse manuelle précédente.

1. Si des exemples japonais apparaissent dans un dictionnaire de proverbes japonais, ils sont annotés comme $\{$ prov $\}$, pour "proverbe".

2. Si la traduction française dans le Cesselin est étiquetée par \{fig\}, l'exemple est annoté comme \{colexp\}, pour "collocation", et si la traduction française dans Cesselin est étiquetée par

21 Veuillez bien m'excuser, mais n'êtes-vous pas monsieur Yamada?

22 Le projet UNL (Universal Networking Language) a été fondé à l'Institut IAS de l'Université des Nations Unies (UNU) à Tokyo en 1996, avec le soutien de l'UNU et le soutien financier de la société ASCII et de l'UNL-IAS, au titre du développement de la technologie de pointe.

http://www.undl.org/unlsys/unl/unl2005/attribute.htm (Uchida, Zhu et Della Senta, 2006)

23 Les lexèmes interlingues (UW) sont créés par des membres d'UNL, qui stockent toute l'information apparentée dans une structure de treillis, où les UW sont reliés par des relations comprenant des relations hiérarchiques (10 niveaux) comme "icl" (une sorte de) et "iof" (une instance de), et signifient la soussignification et la quantité équivalente du mot-clé, respectivement. http://www.undl.org/unlexp/.

24 Le dictionnaire UNL-UW contient à l'heure actuelle, 1269421 mots-vedette pour le japonais, 520305 motsvedette pour le français et 1458686 mots-vedette pour l'anglais. Les attributs sémantiques sont constitués de 58 étiquettes et de 39 étiquettes de relations sémantiques. 
\{botanic\}, zoology\},... , l'exemple est annoté $\{$ domexp $\}$, pour "utilisation spécifique au domaine" indiqué.

3. Si les lexèmes de deux sorties de traduction correspondent à $100 \%$, l'exemple est annoté comme \{ordusg\}, pour "exemple d'utilisation ordinaire".

4. Si les deux sorties de traduction ne sont pas dans les cas 1, 2, 3 ci-dessus, elles sont examinées avec une liste KWIC en utilisant un logiciel, appelé Sketch Engine ${ }^{25}$. Si un exemple apparaît avec certaines occurrences, il est considéré comme une collocation ou un quantificateur (Alda, 2011).

5. Contrairement à la liste KWIC, si un exemple est un syntagme nominal composé de "nombre nom", alors l'exemple est annoté comme \{quant\} pour "quantificateur" (Tomokiyo, Boitet \& Mangeot, 2017), (Miyagawa 1989).

6. Si les exemples dans les deux résultats de traduction n'apparaissent pas dans la liste KWIC avec certaines occurrences, la synonymie entre les mots dans les deux résultats de traduction est examinée en utilisant un dictionnaire UNL-UW.

7. Si le verbe prédicatif et ses actants ${ }^{26}$ dans les deux traductions de l'exemple correspondent, et si les mots du reste des exemples traduits correspondent au niveau de la synonymie, alors l'exemple est annoté comme \{ordusg\} pour "exemple d'usage ordinaire".

8. S'il n'y a pas de correspondance entre les lexèmes contenus dans les deux traductions de l'exemple, il est annoté \{colexp\}, pour "utilisation en collocation", sauf s'ils sont déjà classés comme proverbes, quantificateurs, ou usage spécifique au domaine.

9. Si un exemple n'appartient pas à un des cas $1,2,3,4,5,6$ 7, 8, mentionnés ci-dessus, l'exemple est annoté comme \{ordusg\} pour "exemple d'utilisation ordinaire".

\section{Remarque}

Les exemples dans les dictionnaires ne sont en général pas très longs (environ 10 mots en moyenne), et comprennent en certaines proportions des proverbes, des groupes nominaux, des groupes adjectivaux, ou des phrases incomplètes. Donc, si nous cherchons à appliquer notre méthode de classification/annotation à d'autres documents, nous ne sommes pas sûrs de pouvoir obtenir les mêmes résultats que dans le cas d'un dictionnaire.

\section{Références}

Alda Mari. 2011. Quantificateurs polysémiques, Université Paris-Sorbonne, Vol.23, France.

Mathieu Mangeot-Nagata. 2016. Collaborative construction of a good quality, broad coverage and copyright free Japanese-French dictionary. HAL-01294566.

Mutsuko Tomokiyo, Christian Boitet et Mathieu Mangeot. 2017. Development of a classifiers/quantifiers dictionary towards French-Japanese MT, MTSummit 2017, Japan

Mutsuko Tomokiyo et Christian Boitet. 2016. Corpus and dictionary development for classifiers/quantifiers towards French-Japanese machine translation, COLING, Cog-Alex 2016, Japan

Shigeru Miyagawa. 1989. Structure and case marking in Japanese, Syntax and Semantics, Vol.22, New York.

Uchida, H., Zhu, M., \& Della Senta, T.G. 2006. Universal Networking Language. UNDL Foundation, Japan.

25 Le Sketch Engine est un outil de gestion de corpus ; il contient 400 corpus prêts à l'emploi Nous y ajoutons le corpus de Mathieu Mangeot.

26 Afin de connaître le verbe prédicatif et les actants de chaque exemple traité, nous avons utilisé un analyseur appelé MeCab. http:/taku910.github.io/mecab/ 